\title{
Análisis espectral de las lesiones melanocíticas adquiridas
}

Spectral analysis of acquired melanocytic lesions

\section{Iriana Álvarez¹, Sandra Vivas², Aarón Muñoz ${ }^{3}$}

Grupo de Investigadores, Centro de Investigaciones Médicas y Biotecnológicas, Universidad de Carabobo, Valencia, Venezuela

1. Médica dermatóloga, Ciudad Hospitalaria Dr. Enrique Tejera, Facultad de Ciencias de la Salud, Universidad de Carabobo, Valencia, Venezuela

2. Médica dermatóloga; jefe, coordinadora de posgrado de Dermatología, Universidad de Carabobo, Valencia, Venezuela

3. Licenciado en Física, maestría y doctorado en Ciencias Básicas con especialidad en Óptica; director general, Centro de Investigaciones Médicas y Biotecnológicas, Universidad de Carabobo, Valencia, Venezuela

\section{RESUMEN}

Introducción. A lo largo de la historia se han establecido distintos métodos para obtener información morfológica de las lesiones pigmentadas. La regla de oro para el diagnóstico es la biopsia; sin embargo, se han desarrollado técnicas ópticas no invasivas como la espectrofotometría de reflexión difusa.

Objetivo. Caracterizar las lesiones melanocíticas adquiridas con espectrofotometría de reflexión difusa.

Materiales y métodos. Se hizo un estudio de tipo validación prospectiva, con diseño de cohorte. La población estuvo constituida por 63 pacientes con lesiones pigmentadas adquiridas que acudieron a la consulta del Servicio de Dermatología, Ciudad Hospitalaria Dr. Enrique Tejera. La muestra incluyó 120 nevos clasificados por su diagnóstico clínico e histopatológico.

Resultados. Se evidenciaron diferencias de reflectancia entre cada una de las lesiones estudiadas, y el nevo displásico fue el que presentó mayor absorción de melanina, lo cual se relaciona con que, de estas, es la lesión de mayor profundidad en la piel.

Conclusión. Los nevos melanocíticos estudiados se caracterizan por presentar un espectro definido. Se recomienda incluir la espectrofotometría de reflexión difusa como método accesorio de diagnóstico y seguimiento en la consulta dermatológica.

PALABRAS CLAVE: nevo melanocítico, análisis espectral, biopsia

\section{SUMMARY}

Introduction: History has established different methods for morphological information of pigmented lesions. The gold standard for diagnosis is biopsy; however, noninvasive optical techniques such as diffuse spectrophotometry reflectance have been developed.

Objective: To characterize acquired melanocytic lesions using diffuse spectrophotometry reflectance.

\author{
Correspondencia: \\ Iriana Álvarez \\ Email: \\ iriana47@hotmail.com \\ Recibido: 24/10/2016 \\ Aceptado: 24/04/2017
}

Conflictos de interés:

No se reportan conflictos de interés.

Financiación:

Ninguna. 
Materials and methods: We carried out a prospective study, cohort study. The population consisted of 63 patients with acquired melanocytic lesions who consulted the Service of Dermatology, Ciudad Hospitalaria Dr. Enrique Tejera, Valencia, Venezuela. The sampled included 120 nevi classified clinical and histopathological diagnosis. For data collection a Hunterlab MiniScan XE Plus spectrophotometer was used.

Results: There are differences in reflectance between each of the lesions studied. Dysplastic nevi present greater melanin absorption which is related to a deeper lesion on the skin.

Conclusion: Melanocytic nevi studied are characterized by a defined spectral signature. It is recommended to include diffuse spectrophotometry reflectance, as an optional method of diagnosis and monitoring in dermatologic consultation.

KEY WORDS: Nevus pigmented, spectrum analysis, biopsy

\section{"El diagnóstico clínico de las lesiones pigmentadas en piel se hace generalmente mediante la apreciación visual, empleando métodos como la regla $A B C D E$, el método EFECTIVO y la lista de los tres puntos de verificación".}

\section{INTRODUCCIÓN}

Existen diferentes tipos de nevos melanocíticos en relación con su epidemiologia, morfología, evolución y riesgo de evolución maligna. La posibilidad de un error diagnóstico es preocupante, debido a las consecuencias que se originarían en el paciente cuando estas lesiones corresponden a neoplasias malignas ${ }^{(1-3)}$.

El diagnóstico clínico de las lesiones pigmentadas en piel se hace generalmente mediante la apreciación visual, empleando métodos como la regla $\mathrm{ABCDE}$, el método EFECTIVO y la lista de los tres puntos de verificación ${ }^{(3,4)}$; no obstante, hasta para el dermatólogo experto son técnicas subjetivas y cualitativas. La biopsia aún se mantiene como el método estándar para obtener un diagnóstico confiable; sin embargo, estas son costosas, invasivas y los resultados no se obtienen inmediatamente ${ }^{(5,6)}$.

El incremento en la incidencia de las lesiones cutáneas pigmentadas en la última década, junto con el hecho de que un diagnóstico temprano de las lesiones malignas ayuda a reducir la mortalidad por estas enfermedades, ha fomentado el desarrollo de investigaciones para emplear técnicas ópticas no invasivas en el diagnóstico dermatológico ${ }^{(6,7)}$.

Por consiguiente, el desarrollo de investigaciones sobre el empleo de técnicas, como la dermatoscopia, la videodermatoscopia, la microscopía confocal de re- flectancia y la espectrofotometría de reflexión difusa, abrieron una nueva dimensión en el universo de los nevos melanocíticos ${ }^{(2,5)}$, por medio de la observación de colores y estructuras internas que no son visibles a simple vista y que permiten diagnosticar lesiones y establecer parámetros ópticos en los tejidos biológicos con el fin de obtener un diagnóstico temprano de las lesiones malignas ${ }^{(7,8)}$.

La espectrofotometría de reflexión difusa se basa en el estudio de la incidencia y la reflexión de la luz sobre la piel. Cuando la luz incide sobre la piel, ocurren varios fenómenos ópticos en sus diferentes capas: absorción, reflexión y transmisión. La luz reflejada emerge nuevamente a la superficie y es analizada por el espectrofotómetro, el cual la representa en una curva de reflexión difusa $^{(8,9)}$ (figura 1).

Las estructuras del tejido que absorben la luz se conocen como cromóforos, los cuales absorben ondas de distintas longitudes. Los cromóforos más comunes encontrados en la piel son la melanina y la hemoglobina $^{(8-11)}$. Las propiedades tisulares de absorción y difusión de la luz dependen de su composición bioquímica, su estructura celular y la longitud de onda de la luz incidente. Se ha demostrado que las propiedades ópticas de los tejidos malignos difieren de los de los tejidos sanos y normales ${ }^{(12-14)}$.

Es por ello que la espectrofotometría de reflexión di- 

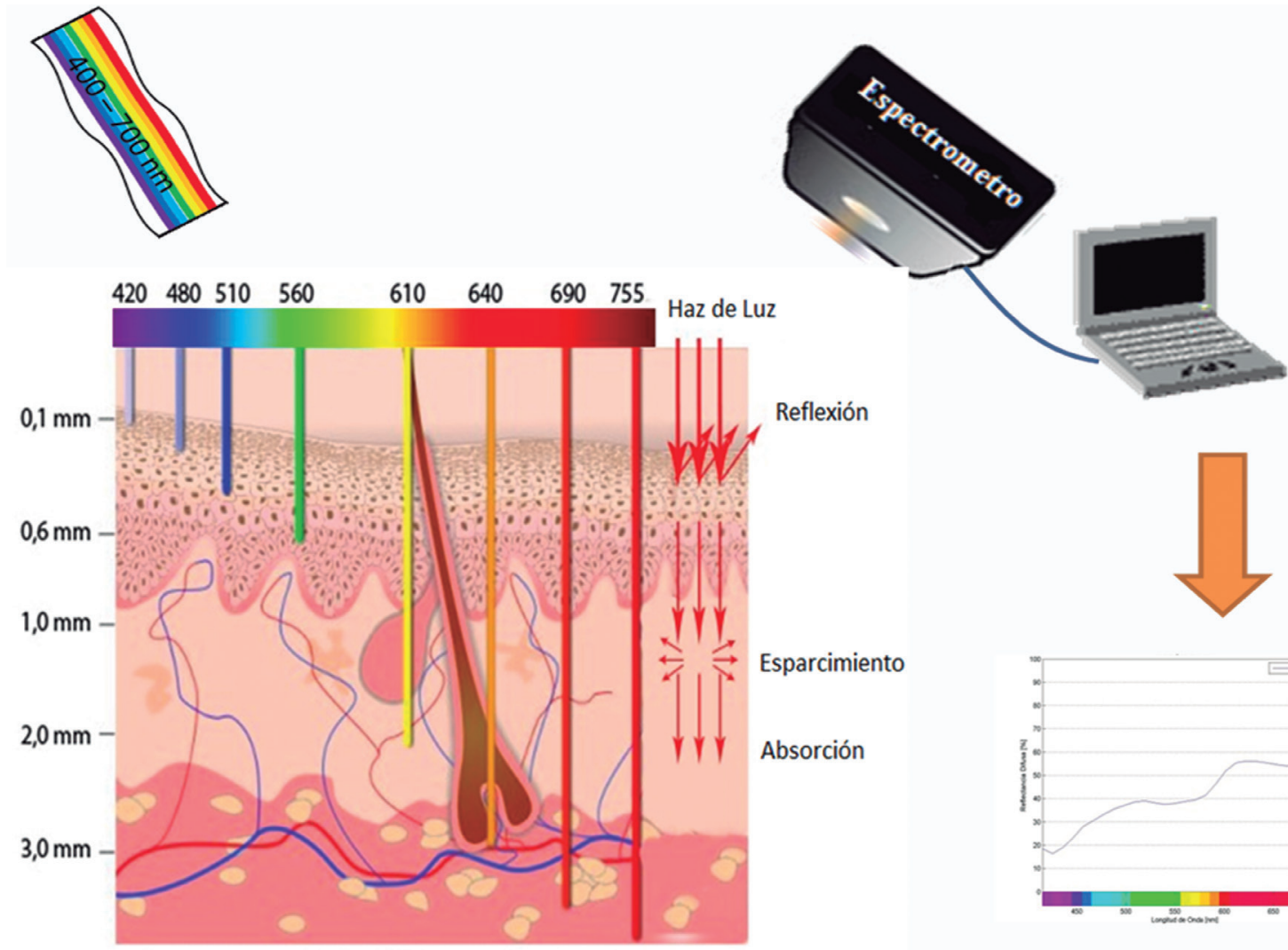

Figura 1. Incidencia de la luz en la piel, captación de la reflexión de la luz por el espectrofotómetro y traducción de la información en una curva espectral por un computadoruente: Centro de Investigaciones Médicas y Biotecnológicas, Universidad de Carabobo (CIMBUC)

fusa representa una herramienta prometedora en el acompañamiento del diagnóstico dermatológico ${ }^{(15,16)}$. Además, el impacto sobre la observación directa de los nevos abre una ventana a la comprensión de la evolución y los factores que influyen en sus diferentes patrones ${ }^{(17-19)}$

Con el fin de mejorar la precisión diagnóstica de las lesiones melanocíticas, las cuales son uno de los más frecuentes motivos de consulta en el Servicio de Dermatología, de la Ciudad Hospitalaria "Dr. Enrique Tejera” (el mayor centro de referencia dermatológica en el centro occidente del país), nos planteamos caracterizarlas con espectrofotometría de reflexión difusa, determinar las diferencias de reflexión y absorción de los cromóforos en las lesiones estudiadas y contrastar las curvas espectrales con el diagnóstico histopatológico.

\section{MATERIALES Y MÉTODOS}

Se llevó a cabo un estudio de tipo validación prospectiva ${ }^{(20)}$, de diseño de cohorte, el cual permitió evaluar la capacidad predictiva diagnóstica de la técnica. Se controlaron los principales sesgos, ya que se llevó a cabo en sujetos sin diagnóstico previo y después de practicar la espectrofotometría de reflexión difusa en validación, y se siguió hasta alcanzar el diagnóstico definitivo mediante la histopatología.

La población estuvo constituida por todos los pacientes con lesiones pigmentadas melanocíticas adquiridas que acudieron a la consulta del Servicio de Dermatología, Ciudad Hospitalaria "Dr. Enrique Tejera”, en el período comprendido abril y agosto de 2015. La muestra incluyó 120 nevos, clasificados según el diagnóstico en los cuatro tipos de nevos melanocíticos adquiridos ${ }^{(2,3)}$. Después del diagnóstico clínico, se hizo la medición espectral (piel sana perilesional y centro de la lesión) y, por último, la toma de la biopsia. 
El protocolo clínico de este estudio fue revisado y aprobado por el Comité de Ética del hospital Ciudad Hospitalaria “Dr. Enrique Tejera”. Se le explicó a cada paciente en qué consistían las medidas espectrofotométricas y se obtuvo el consentimiento escrito de cada uno de ellos antes de la medición.

Para obtener los valores de reflectancia de las lesiones cutáneas pigmentadas, se utilizó un espectrofotómetro de reflexión difusa marca Hunterlab, modelo MiniScan con esfera integradora de 8 grados y con un rango espectral de 400 a $700 \mathrm{~nm}$ con una resolución de $10 \mathrm{~nm}$, el cual está constituido por una fuente de luz blanca y un dispositivo monocromático (figura 2).

La información obtenida se transfirió a un computador que tiene un software universal Hunterlab 10.0 basado en Java, bajo el sistema operativo Windows 7.0, el cual permitía una rápida medición de los datos espectrales, su visualización y su almacenamiento. Asimismo, se graficó de forma lineal la información en un eje de coordenadas; en el eje horizontal, se representaron las distintas longitudes de onda en un rango de 400 a 700 $\mathrm{nm}$ y, en el eje vertical, el porcentaje de la reflectancia de la luz sobre la piel. Posteriormente, con esta infor- mación se elaboraron curvas para su análisis.

Una vez obtenidas las curvas espectrales para cada una de las lesiones melanocíticas, se procedió a incluir la información en un sistema de procesamiento de datos que permitió obtener el promedio espectral (porcentaje de reflectancia) de cada lesión y, posteriormente, compararlo con la clasificación histopatológica de la misma. El análisis se hizo mediante el programa Microsoft Office Excel para Windows 8.

Se diseñó un instrumento que permitió registrar los datos clínicos y las mediciones espectrales de cada paciente. Los datos fueron registrados y almacenados en un formato electrónico de base de datos (ACCESS) y de hoja de cálculo (EXCEL). Los resultados se analizaron por medio de estadística descriptiva con base en el análisis de frecuencias absolutas y relativas, y se presentan en tablas y gráficos.

\section{RESULTADOS}

Se estudiaron 120 lesiones melanocíticas de 63 pacientes, clasificadas de acuerdo con el diagnóstico

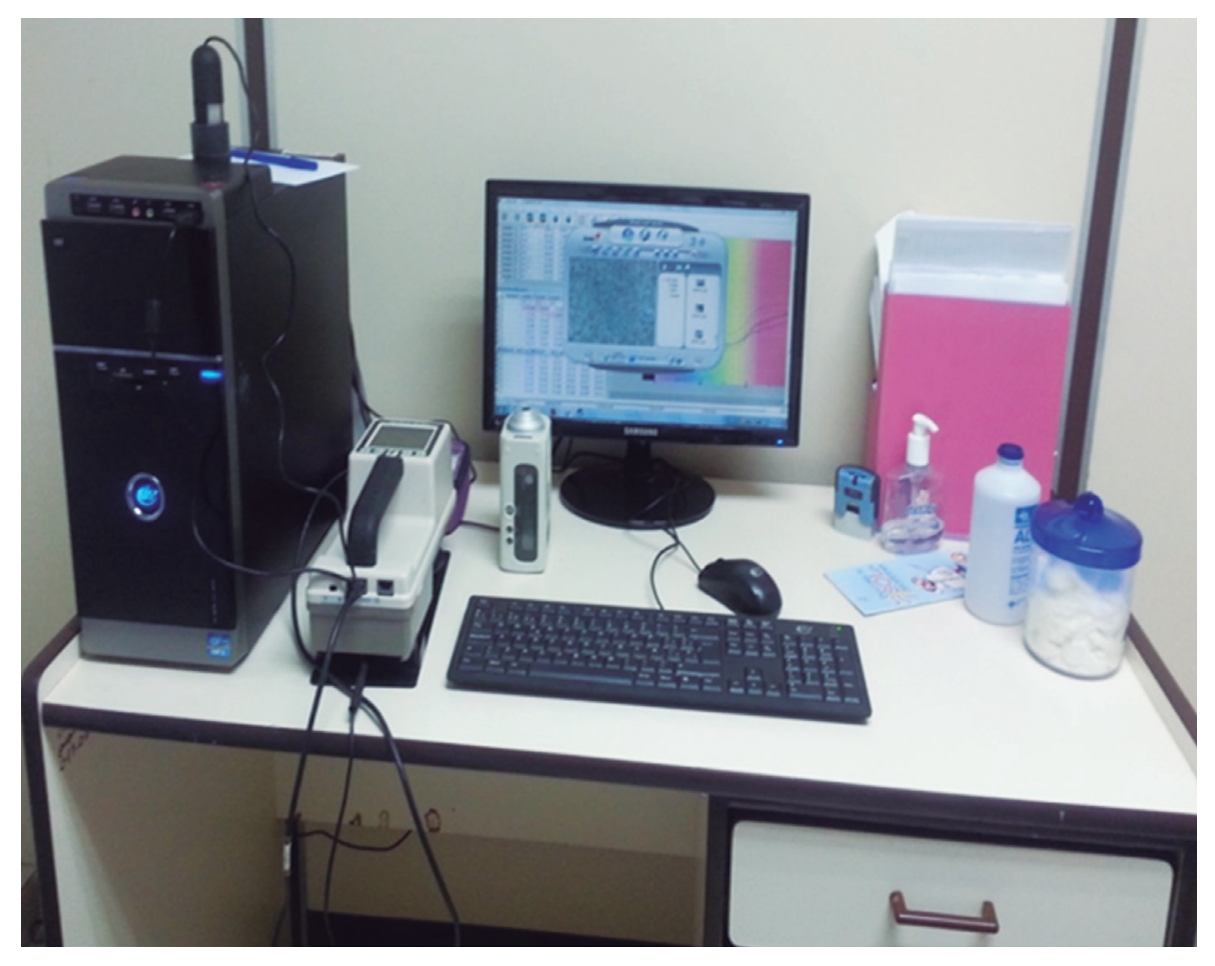

Figura 2. Equipo de espectrofotometría de la Unidad de Fotodiagnóstico. Servicio de Dermatología, Ciudad Hospitalaria Dr. Enrique Tejera. Fuente: Unidad de Fotodiagnóstico CHET 
clínico y el histopatológico (figura 3). Los nevos melanocíticos se distribuyeron según el diagnóstico histopatológico; predominó el nevo intradérmico (31,6 \%), seguido, en orden de frecuencia, por el nevo de unión $(29,1 \%)$, el nevo compuesto $(24,1 \%) \mathrm{y}$, con la menor frecuencia, el nevo displásico (15\%) (figura 4).

Para explicar este hecho, se representa la curva correspondiente al nevo de unión como una curva ascendente con reflectancias entre 20 y $23 \%$, y un ascenso con un pico en $600 \mathrm{~nm}$ (con reflectancia de 23 a $52 \%$ ) (figura 5). En el estudio de histopatología, el nevo de unión es la lesión más superficial de la piel, localizada en la unión dermoepidérmica.

En las curvas espectrales del nevo intradérmico y del compuesto, se observaron similitudes con reflectancias de 15 a $20 \%$ y de 14 a $17 \%$, respectivamente, entre los 550 y los $600 \mathrm{~nm}$; hacia los $600 \mathrm{~nm}$, se obtuvo una curva más cercana a la línea basal, con reflectancias máximas de 40 \% (figuras 6 y 7). Desde el punto de vista histopatológico, el nevo compuesto se presenta tanto en la unión dermoepidérmica como en la dermis; por otro lado, el nevo intradérmico tiene una localización más profunda en la dermis.

Por último, el nevo displásico generó una curva casi aplanada y las menores reflectancias, de 10 a $11 \%$, en las longitudes de onda donde se evidencian los mayores cambios correspondientes a la melanina; hacia los $600 \mathrm{~nm}$, las reflectancias máximas fueron de $29 \%$ (figura 8). En la histopatología, las tecas o las agrupaciones de melanocitos en este tipo de lesiones se localizan a mayor profundidad que en el resto de los nevos estudiados.

\section{DISCUSIÓN}

Para constatar las diferencias espectrales entre cada tipo de lesión melanocítica, se tomó como referencia la curva espectral del fototipo cutáneo IV, que es el más común en nuestra población. Esta curva presenta dos mínimos (valles), relacionados con la absorción de la hemoglobina (400 $\mathrm{nm}$ ), y una meseta con cierto grado de inclinación, dada por la absorción de la melanina $(600 \mathrm{~nm})$, hallazgos que corresponden a las características ya reportadas en los estudios presentados por Pardo, et al. ${ }^{(21)}$, y Martínez, et al. ${ }^{(22)}$.

Se observaron semejanzas en el comportamiento de los cromóforos en los cuatro tipos de lesiones estudiadas. Los puntos de absorción para la hemoglobina estuvieron en el rango de longitudes de onda de 400 a 420 $\mathrm{nm}$ y, los de la melanina, entre 550 y $600 \mathrm{~nm}$, siendo este punto donde se observaron las mayores diferencias.

Para explicar mejor los resultados, es importante resaltar que el estudio de la melanina es de importancia capital al momento de diferenciar cada una de las curvas espectrales, ya que esta rige la forma de la curva. La profundidad de penetración de la melanina en las capas de la piel, determina los diferentes tipos de nevos melanocíticos. La melanina se puede clasificar en dos clases: la eumelanina, de color negro-marón, y la feomelanina, de color amarillo-rojo; ambas son polímeros con unidades de monómeros múltiples unidas por enlaces no hidrolizables.

Estos polímeros tienen una característica importante con una amplia absorción desde los $200 \mathrm{~nm}$ en toda la
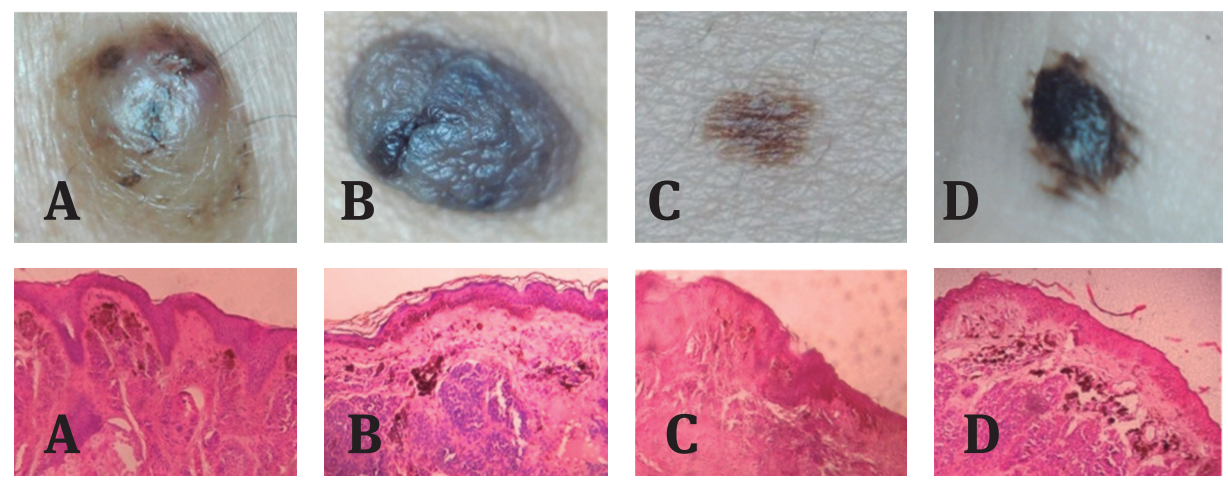

A. Nevus intradérmico

B. Nevus compuesto

C. Nevus de unión

D. Nevus displásico

Figura 3. Clasificación clínica e histopatológica de lesiones pigmentadas melanocíticas adquiridas. Fuente: Unidad de Fotodiagnóstico CHET 
Tipos de Nevus

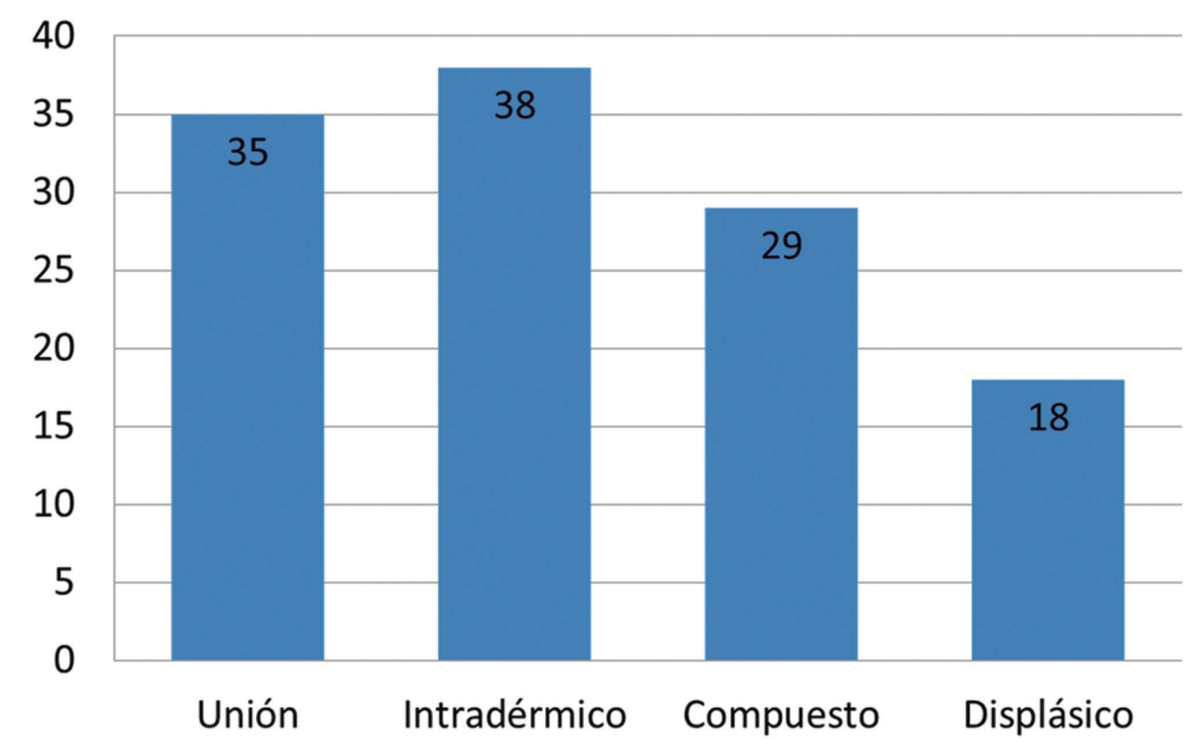

Figura 4. Distribución de nevos melanocíticos según el diagnóstico histopatológico Fuente: Unidad de Fotodiagnóstico CHET

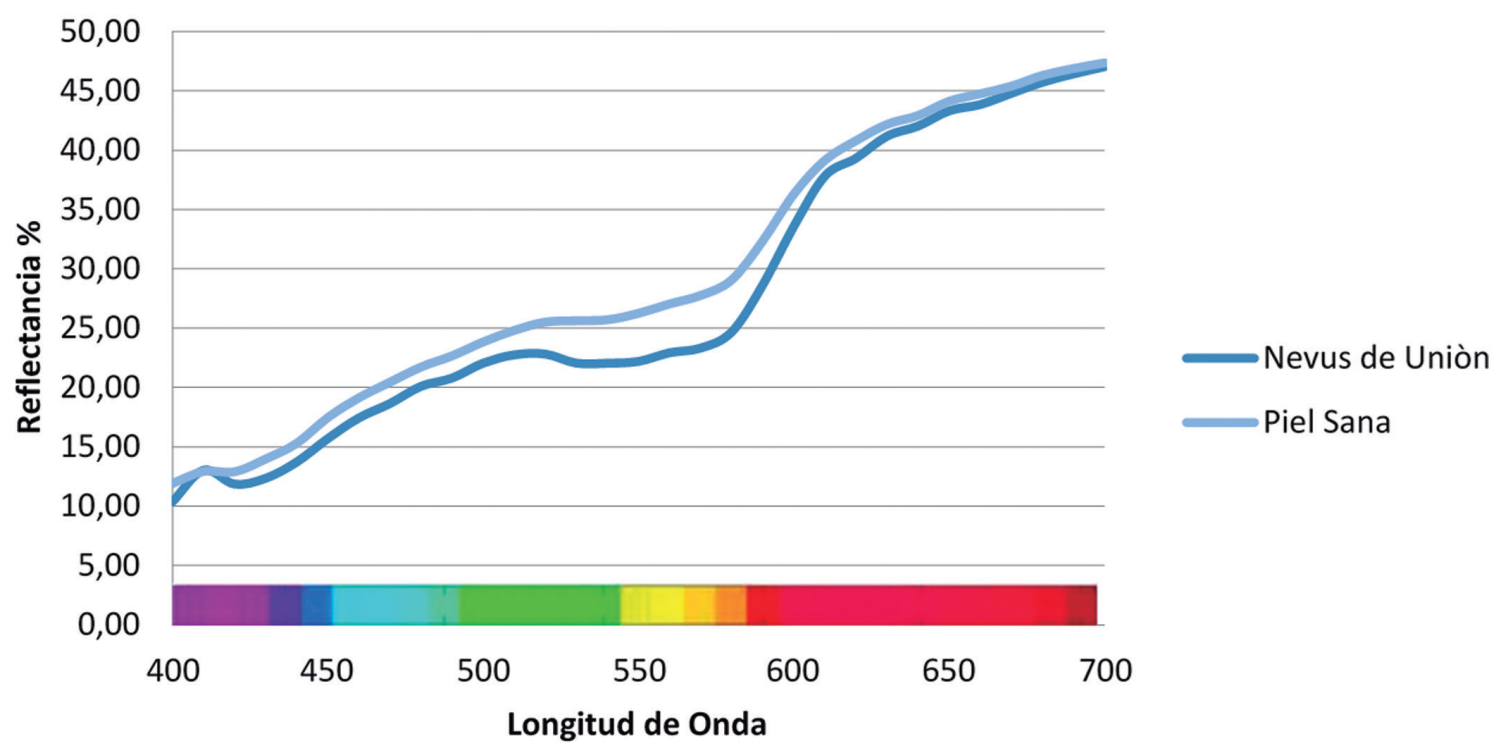

Figura 5. Curva espectral de nevos melanocíticos de unión Fuente: Unidad de Fotodiagnóstico CHET 




Figura 6. Curva espectral de nevos melanocíticos intradérmicos Fuente: Unidad de Fotodiagnóstico CHET



Figura 7. Curva espectral del nevos melanocíticos compuestos Fuente: Unidad de Fotodiagnóstico CHET 


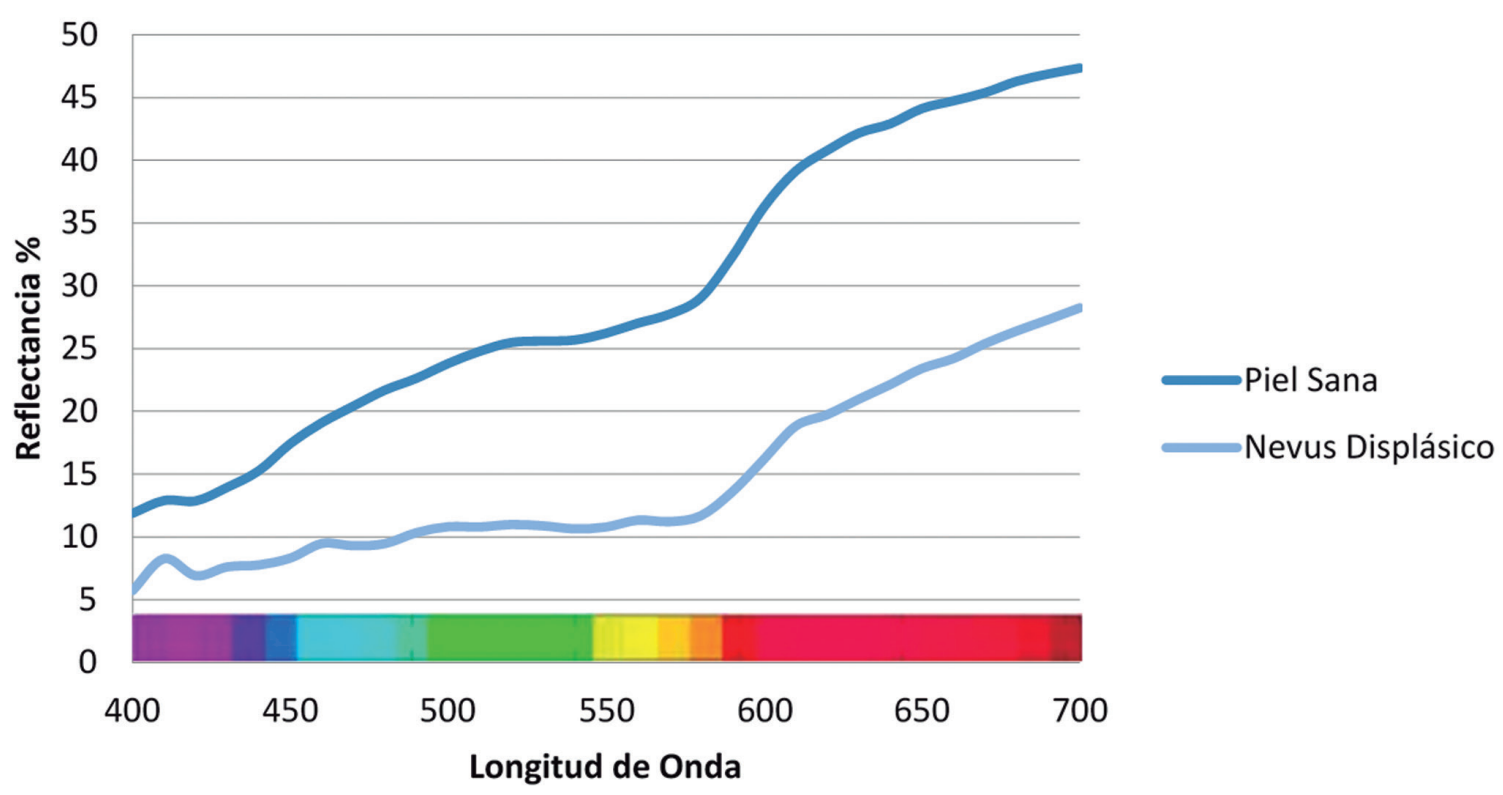

Figura 8. Curva espectral del nevos melanocíticos displásicos Fuente: Unidad de Fotodiagnóstico CHET

longitud, hasta más de $1.000 \mathrm{~nm}^{(23)}$. La curva correspondiente a la absorción en el espectro visible de este cromóforo, se presenta en la figura 9.

Cuando existe una mayor capacidad de absorción del rango espectral utilizado, la curva es más cercana a la linea de base y existe un menor porcentaje de reflectancia. Pardo, et al. ${ }^{(21)}$, informaron hallazgos similares y señalaron que la melanina es un compuesto que absorbe luz y radiación ultravioleta, y, por lo tanto, es lógico pensar que existe una relación directamente proporcional: a mayor cantidad de melanina, mayor absorción de luz.

La profundidad de penetración de la melanina en las capas de la piel conlleva lesiones que van desde los diferentes tipos de nevos, hasta lesiones malignas como el melanoma. Para poder dar explicación de los hallazgos encontrados en las curvas espectrales de los nevos melanocíticos adquiridos, es necesario contrastarlos con los hallazgos histopatológicos, teniendo en cuenta que la caracterización de estas lesiones va a estar determinada por la localización de las 'tecas' de melanina en las diferentes capas de la piel ${ }^{(1)}$.

El hecho de que los nevos de unión se caracterizan por concentraciones de 'tecas' de melanocitos en la unión dermoepidérmica (por ello se consideran precursores de todos los nevos), explica que las curvas de reflectancia de estos nevos mostraran los mayores valores de reflectancia, en comparación con el resto de las lesiones estudiadas.

A pesar de las similitudes entre el nevo compuesto y el intradérmico, este último presentó una menor reflectancia en el espectro de reflexión de la melanina de-

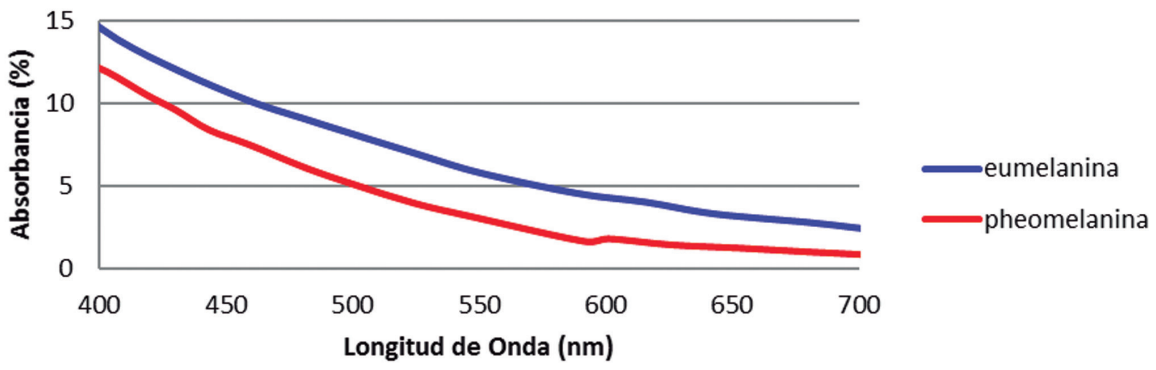

Figura 9. Curva espectral de la absorción de la melanina: eumelanina y feomelanina. Fuente: CIMBUC 2015 
bido a su mayor profundidad en la piel.

A diferencia del presente estudio, en los de Cordo, et al. ${ }^{(9)}$, y de Zonios, et al. ${ }^{(17)}$, quienes compararon las lesiones pimentadas y la piel sana mediante espectrofotometría, no se obtuvo ninguna diferencia apreciable entre los nevos compuestos y los intradérmicos.

Se sabe que el nevo displásico es la lesión melanocítica de mayor profundidad, lo cual explica la mayor absorción de la melanina en el rango de longitud de onda de 550 a $600 \mathrm{~nm}$, generando una menor pendiente de la curva en comparación con las otras lesiones estudiadas, lo cual puede relacionarse con la presencia de atipias celulares.

El estudio del aumento de las concentraciones de melanina y la profundidad de las lesiones névicas a partir de las curvas de reflectancia difusa, permitiría tener un elemento de juicio para la transición histológica de nevo común a nevo displásico y melanoma, lo cual concuerda con el estudio de García, et al. ${ }^{(19)}$, en el 2011, en el cual determinaron que la absorción de la melanina es mayor en los nevos displásicos y los melanomas relacionados con lesiones benignas. Por otro lado, Zonios, et al. ${ }^{(17)}$, en el 2008, reportaron evidencias de que la transición histológica de nevo displásico a melanoma in situ se refleja en el espectro de absorción de la melanina.

Las limitaciones en el presente estudio fueron: no poder aumentar el número de casos por la disponibilidad del equipo y el procesamiento de las biopsias; no disponer de una mayor resolución en el espectrofotómetro utilizado, y el desconocimiento, en general, de los especialistas sobre la aplicación de la técnica.

\section{CONCLUSIONES Y RECOMENDACIONES}

Los nevos melanocíticos estudiados se caracterizan por presentar un espectro definido. Asimismo, hubo correspondencia entre la localización de 'tecas' de melanocitos en el estudio histopatológico y los valores de reflectancia, en cada uno de los nevos estudiados.

La espectrofotometría de reflexión difusa se vislumbra como una técnica no invasiva de control y seguimiento de las lesiones melanocíticas; como recomendación del estudio consideramos que con esta técnica se pueden realizar barridos de despistaje de lesiones névicas, aumentando la cobertura y el impacto en los sistemas de salud para realizar despistajes en tiempo real a distancia remota. Se sugiere realizar más estudios implementando esta técnica óptica en lesiones melanocíticas a fin de demostrar su especificidad y sensibilidad.

\section{REFERENCIAS}

1. Rex J, Ferrandiz C. Nevus melanocíticos. Fecha de consulta: 4 de febrero de 2015. Disponible en: https://www.aeped.es/sites/default/files/documentos/nevus.pdf

2. Friedman R. Rigel DS, Kopf AW. Early detection of malignant melanoma: The role of physician examination and self examination of the skin. CA Cancer J Clin. 1985;35:130-51.

3. Wolf K, Johnson R. Color atlas \& synopsis of clinical dermatology. Buenos Aires: McGraw Hill; 2009. p. 178-91.

4. Wolff K, Goldsmith L, Katz S, Gilchrest B, Paller A, Leffell D. Fitzpatrick Dermatología en Medicina General. Séptima edición. Buenos Aires: Editorial Panamericana; 2010. p. 1099-133.

5. Garnacho GM, Moreno JC. Trastornos de la pigmentación: lentigos, nevus y melanoma. Fotoprotección. Servicio de Dermatología. Hospital Universitario Reina Sofía de Córdoba. Pediatr Integral. 2012;XVI: 321-31.

6. Lacarrubba F. Use of videodermatoscopy in dermatology. En: Berardesca E, Maibach H, Wilheim K, editores. Non Invasive Diagnostic Techniques in Clinical Dermatology. Berlin: Springer; 2014. p. 3-26.

7. Bono A, Tomatis S, Bartoli C, Tragni G, Radaelli G, Maurichi A, et al. The ABCD system of melanoma detection. A spectrophotometric analysis of the asymmetry, border, color and dimension. Cancer, 1999;85:72-7.

8. Fodor L, Ullmann Y, Elman M. Aplicaciones estéticas de la luz pulsada intensa. Israel. Amolca; 2012. p. 11- 20

9. Cordo M, Sendra J, Viera A, Santana A, López SM. Diferenciación de piel sana y lesiones cutáneas pigmentadas mediante espectroscopía de reflectancia óptica difusa. Óptica Pura y Aplicada. 2006;39:341-54.

10. Orozco E. Estudio de lesiones en piel mediante espectroscopía de reflexión difusa (tesis). Puebla, México: Instituto Nacional de Astrofísica, Óptica y Electrónica; 2009.

11. Tomatis S, Bono A, Bartoli C, Carrara M, Lualdi M, Tragni G, et al. Automated melanoma detection: multispectral imaging and neural network approach for classification. Phys Med. 2003;30:21221.

12. Marchesini R, Brambilla M, Clemente C, Maniezzo $\mathrm{M}$, Sichirollo AE, Testori A, et al. In vivo spectrophotometric evaluation of neoplastic and nonneo- 
plastic skin pigmented lesions. III. CCD camerabased reflectance imaging. Photochem Photobiol. 1995;62:151-4.

13. Scarisbrick J, Pickard C, Lee A, Briggs G, Johnson $\mathrm{K}$, Bown S, et al. Elastic scattering spectroscopy in the diagnosis of pigmented lesions: Comparison with clinical and histopathological diagnosis. Proceedings of SPIE 2003,5141:147-56.

14. Marchesini R, Cascinelli N, Bambrilla M, Clemente C, Mascheroni L, Pignoli E, et al. In vivo spectrophotometric evaluation of neoplastic and nonneoplastic skin pigmented lesions. II. Discriminant analysis between nevus and melanoma. Photochem Photobiol. 1992;55:515-22.

15. Orozco E, Iruretagoyena G, Vázquez S, Delgado J, Castro J, Gutiérrez F. Métodos de clasificación para identificar lesiones en piel a partir de espectros de reflexión difusa. Revista Ingeniería Biomédica. 2010;4:34-40.

16. Ascierto P, Palla M, Ayala F, De Michele I, Caracò C, Daponte A, et al. The role of spectrophotometry in the diagnosis of melanoma. 2010;10:5.

17. Zonios G, Dimou A. Optical properties of human melanocytic nevi in vivo. Photochem Photobiol. 2009;85:298-303.

18. Feinsilber D, Acosta A, Rosati O, Kogan N Schroh $\mathrm{R}$, Corbella C. Método de codificación de lesiones pigmentarias. Dermatol Argent. 2014;20:94-8.

19. García A, Smith E, Zou J, Duvic M, Prieto V, Wang LV. In-vivo characterization of optical properties of pigmented skin lesions including melanoma using oblique incidence diffuse reflectance spectrometry. J Biomed Opt. 2011;16:020501.

20. Carnero-Pardo C. Evaluación de las pruebas diagnósticas. Rev Neurol. 2005;40:641-3.

21. Pardo E, Martínez C, Álvarez I, Vivas S, Muñoz A. Método diagnóstico no invasivo en dermatología: espectrofotometría de reflexión difusa. Informe Médico. 2015;17:49-54.

22. Martínez C, Pardo E, Álvarez I, Muñoz A, Vivas S. Determinación espectrofotométrica del eritema en psoriasis. Rev Asoc Colomb Dermatol. 2015;23:250-5.

23. Rox A, Parrish J. The optics of human skin. J Invest Dermatol. 1981;77:13-9. 